\title{
An Observational Study to Determine and Compare Effects of Various Physical Test Components on BMI of School Going Children
}

\section{Jhaveri B* and Savaliya Gr}

Shrimad Rajchandra College of Physiotherapy, Uka Tarsadia University, India

*Corresponding author: Bhavik Jhaveri, Uka Tarsadia University, Tal.-Bardoli, Dist.Surat, Gujarat, India, Tel: +91-9913752456; Email: bhavik.jhaveri@utu.ac.in

\section{Research Article \\ Volume 1 Issue 2}

Received Date: September 28, 2018

Published Date: October 25, 2018

DOI: $10.23880 /$ aphot- 16000107

\section{Abstract}

Purpose: To evaluate and compare the physical fitness components in relation to Body Mass Index of school going children.

Study design: Across-sectional observational study.

Methods: 100 school going children of 8th and 9th grade were selected to serve as subject for this study. Children were divided into three groups by calculating BMI as per the height and weight of each child, I.e. Low BMI (N=35), Middle BMI $(\mathrm{N}=35)$ and High BMI $(\mathrm{N}=30)$ groups, which were compared with each other. All the subjects were tested on Curl-up test, Shuttle run test, Sit \& Reach test and BMI (Body Mass Index). In order to find out the significance of differences between the three BMI groups of children, mean, standard deviation and p-value were computed.

Results: The results of the study revealed significant differences $(\mathrm{p}<0.05)$ between Low BMI and High BMI groups in their Curl-up test, Shuttle run test and Sit \& Reach test.

Conclusion: low BMI group children were found to be better in muscular strength, flexibility and endurance level compared to middle BMI group \& high BMI group children.

Keywords: Body Mass Index; Physical Fitness Components

\section{Introduction}

Physical fitness is the most important factor in development of physical responses to exercises during the childhood years. Lack of exercise is directly related to various health problems like obesity, hypertension, or cardiovascular diseases [1]. Regular physical activity in children improves physical fitness, which helps in prevention of childhood obesity. Obesity is one of the most prevalent nutritional disease in children \& adolescents in India. Obesity is a medical condition in which excess body fat has accumulated to the extent that it may have a negative effect on health. The prevalence of obesity in children has been increasing in last few decades especially in developing countries like India, this ratio has increased to $8 \%-12 \%$ for boys and $8 \%-13 \%$ for girls [2]. Excess body weight in childhood and adolescence is 


\section{Annals of Physiotherapy \& Occupational Therapy}

associated with a higher risk of developing noncommunicable diseases such as diabetes at younger age.

The specific causes of overweight and obesity in childhood is complex, but despite the consideration of genetic \& physiologic aspects a reduction in physical activities, an increased amount of time devoted to sedentary lifestyle plays crucial role in the development of obesity. People are generally considered obese when their Body Mass Index (BMI), is over $30 \mathrm{Kg} / \mathrm{m}^{2}$, with the range $25-30 \mathrm{Kg} / \mathrm{m}^{2}$ defined as overweight. Obesity increases the likelihood of various health problems particularly heart diseases, Type 2 diabetes, certain type of cancer \& osteoarthritis in later stage of life [3]. Body mass index (BMI) is a statistical measurement derived from height \& weight of an individual. It is the most widely used tool in evaluating a person's health status.BMI value is used to determine whether the individual has acceptable body weight, overweight or obese. BMI value from 15-25 represents no excess mortality risk and over 40 a high risk of great mortality [4]. BMI can be calculated by following equation:

$$
\text { BMI = weight }(\mathrm{kg}) / \mathrm{height}\left(\mathrm{m}^{2}\right)
$$

The most common cause of obesity is excessive food intake, lack of physical activity \& genetic susceptibility [5].

It has been noted that good cardiovascular endurance, muscle strength \& flexibility have a preventive role in childhood obesity [6]. Various physical tests have been suggested in order to improve cardiovascular fitness, muscular strength \& flexibility. These physical tests are 20-m shuttle run test to assess cardiovascular fitness, curl-up test to assess muscular strength and sit \& reach test to assess muscular flexibility of an individual.

Aerobic (cardiovascular) fitness is one of the most important component of physical fitness. Cardiovascular fitness is measured as the amount of oxygen transported in the blood \& pumped by the heart to working muscles. Increasing cardiovascular fitness means increasing the capability of the heart \& the rest of the cardiovascular system to supply oxygen \& energy to your body. The $20-\mathrm{m}$ shuttle run test is simple, easy to administer \& not too time-consuming, it requires minimal equipment, and a large number of individuals can be tested simultaneously [7].

Muscular strength refers to the ability of contractile tissue to produce tension $\&$ a resultant force based on the demands placed on the muscle. The abdominal/trunk region have been selected as areas for testing because of their perceived relationship to activities of daily living, correct posture \& well-functioning back. The curl-up test is a safer \& more effective test since it does not involve the assistance of hip flexor muscle \& minimizes compression in the spine.

Muscular flexibility is defined as ability to move a single joint or series of joints smoothly \& easily through an unrestricted, pain-free ROM. Flexibility allows the participant to move through a full range of motion. The sit $\&$ reach test is a common measure of flexibility, and specifically measures the flexibility of the lower back and hamstring muscles. It is an easy and quick test to perform $\&$ not too time consuming, it requires minimal equipment.

Thus the objective of this study was to evaluate and compare the physical fitness and BMI of school going children.

\section{Materials \& Methods}

Source of data: SDA High school, Surat

Study design: An observational study

Sample size: 100 school going children of $8^{\text {th }} \& 9^{\text {th }}$ standard

\section{Participants}

100 school going children who volunteered to participate in this study, were randomly selected to serve as subjects for this study. All the subjects were selected from the $8^{\text {th }}$ and $9^{\text {th }}$ grade. The subjects were included in this study with age group of 13 to 15 years. (Mean age $13.90 \pm 0.77 \mathrm{yrs}$, mean height $1.53 \pm 0.09 \mathrm{~m}^{2}$, mean weight $49.84 \pm 12.98 \mathrm{~kg}$ and mean BMI $20.9 \pm 4.51 \mathrm{~kg} / \mathrm{m}^{2}$ ).

\section{Inclusion criteria}

Children between 13-16 year

No history of lower extremity injuries

No history of medical illness since past 6 month

No h/o heart disease

\section{Exclusion criteria}

History of back pain

Children with fever or cold on the day of data collection

Children without signed consent form

Any cardiovascular disease

\section{Procedure}

Testing procedure started after getting ethical clearance from the ethical committee of the college as well as after taking permission from the principal of 


\section{Annals of Physiotherapy \& Occupational Therapy}

school. The subjects were explained about the purpose of study in the presence of their school physical education teachers and principal to elicit active cooperation from the subjects. Informed consent was given to all the subjects which need to be signed by their parents. Subjects are recruited in the study after obtaining consent form from the children. All the subjects were tested on Curl-ups, Sit \& Reach test, Beep test and BMI. All the tests were administered at M.N.J. Patel boys' high school and S.D.A. girls' high school playground.

Beep testis a commonly used maximal running aerobic fitness test in which subject has to run between two lines drawn at a distance of $20 \mathrm{~m}$. The test subjects stand behind one of the lines facing the second line, \& begin running when instructed by the audiotape. The speed at the start is quite slow. The subject continues running between the two lines, turning when signaled by recorded beeps. After about one minute, a sound indicates an increase in speed \& the beeps will be closer together. This continues each minute. If the line is not reached in time for each beep, the subject must run to the line turn \& try to catch up with the pace within 2 more "beeps". Also, if the line is reached before the beep sounds, the subjects must wait until the next beep sounds. The test is stopped if the subject fails to reach the line (within 2 meters) for two consecutive ends. Scoring will be done on the level \& number of shuttles $(20 \mathrm{~m})$ reached before they are unable to keep up with recording.

The sit \& reach test is a common measure of flexibility which specifically measures the flexibility of lower back \& hamstring muscles. This test involves sitting on the floor with legs stretched out straight ahead. Shoes should be removed. The soles of feet are placed flat against the box. Both knees should be extended. Then, subjects reaches forward along the measuring line as far as possible. Ensure that the hands remain at the same level, not one reaching further forward than the other. After some practice reaches, the subject reaches out \& holds that position for at 1-2 sec while distance is recorded. Make sure there is no jerky movement. The score is recorded to the nearest centimeter $(\mathrm{cm})$ or half inch as the distance reached by the hand.

The curl-up test measures abdominal strength \& endurance which are important in back support \& core stability .The subject begins this test by lying on their back, knee bent at approximately 140 degrees, feet flat on the floor, legs slightly apart, arms straight \& parallel to the trunk with palms of hands resting on the mat. Keeping heels in contact with the mat, the student curl up slowly, sliding their fingers across the measuring strip until the fingertips reach the other side, then curls back down until their head touches the mat. Scoring will be done by recording the total number of Curl-ups up to maximum of 75 in a minute. The test is also stopped if the student has 2 technique warnings. If the heels come off the floor, the head does not return to the mat, or fingertips do not reach the far side of the measuring strip.

\section{Statistical analysis}

In order to find out the significant difference between different BMI groups, all analysis were performed using the Graph pad Instat Demo (version 3.06). The MannWhitney test was used to find intergroup differences between two BMI groups and the level of significance was set as $\mathrm{p} \leq 0.05$.

\section{Results}

To verify the hypothesis, $Q_{1}$ and $Q_{3}$ of BMI were calculated. Q1 score of BMI was 18.35 \& Q3 score of BMI is 24.9. The entire 100 sample are divided into 3 groups on basis of calculated quartile deviations (Q1 \& Q3). Subjects having BMI values between $\mathrm{Q} 1 \& \mathrm{Q} 3$ were put in the middle group $(\mathrm{N}=35)$. Subjects possessing $\mathrm{BMI}$ values more than Q3 were put in the high BMI group $(\mathrm{N}=30)$ while those having BMI values less than Q1 were assigned to the low BMI (N=35).

In order to find out the significance of differences between low BMI, middle BMI and high BMI of school going children means, standard deviation sand $p$-value were computed. The level of significance was set as $p \leq 0.05$ and data pertaining to this have been presented in Tables 1 to 4 .

It is evident from table 2, that there is a statistically significant difference found between middle BMI \& high BMI groups on curl-up test, sit \& reach test and beep test components of physical fitness, as obtained p-values of $<0.0001,0.008 \& 0.0002$ respectively, which was significant.

It is evident from table 3 , that there is a statistically significant difference found between low BMI \& high BMI groups on curl-up test, sit \& reach test and beep test components of physical fitness, as obtained p-values of $<0.0001,0.002 \&<0.001$ respectively, which was significant.

It is evident from table 4 , that there is statistically significant difference found between low BMI \& middle BMI on curl-up test \& beep test components of physical
Jhaveri B and Savaliya G. An Observational Study to Determine and Compare Effects of Various Physical Test Components on BMI of School Going Children. Ann Physiother Occup Ther 2018, 1(2): 000107.
Copyright@ Jhaveri B and Savaliya G. 
fitness whereas non-significant difference was found on sit \& reach test, as obtained p-value for curl-up test \& beep test was $<0.0001$ and 0.0003 respectively which was significant and p-value for sit \& reach test was 0.51 which was non-significant.

\begin{tabular}{|c|c|c|c|c|c|c|}
\hline & \multicolumn{2}{|c|}{ Low BMI } & \multicolumn{2}{c|}{ Middle BMI } & \multicolumn{2}{c|}{ High BMI } \\
\cline { 2 - 6 } & Mean & SD & Mean SD & SD & Mean SD & SD \\
\hline Curl-up Test (per minute) & 29.68 & 3.96 & 23.51 & 6.87 & 15.76 & 5.45 \\
\hline Sit \& Reach Test (cm) & 3.62 & 5.55 & 4.42 & 8.03 & -0.7 & 5.74 \\
\hline Beep Test (m) & 3.68 & 1.34 & 2.78 & 0.84 & 1.97 & 0.6 \\
\hline
\end{tabular}

Table 1: Descriptive statistics of Body mass Index (BMI) on various components of physical fitness of school going children.

\begin{tabular}{|c|c|c|c|c|}
\hline Physical Fitness Component & BMI Groups & MEAN & SEM & \multirow{2}{*}{ P-Value } \\
\hline $\begin{array}{c}\text { Curl-up } \\
\text { (per minute) }\end{array}$ & Middle & 23.51 & 1.15 & \multirow{2}{*}{$<0.0001$} \\
\hline \multirow{2yyyn}{*}{$\begin{array}{c}\text { Sit \& Reach } \\
\text { (cm) }\end{array}$} & High & 15.76 & 0.99 & \multirow{2}{*}{0.008} \\
\hline \multirow{2yyyn}{*}{ Beep (m) } & Middle & 4.42 & 1.35 & \multirow{2}{*}{0.0002} \\
\cline { 2 - 4 } & High & -0.7 & 1.05 & 0.14 \\
\hline
\end{tabular}

Table 2: Intergroup comparison of Middle BMI and High BMI of school going children on various physical fitness components.

Significant $=p \leq 0.05$, Non-significant $=p>0.005$

\begin{tabular}{|c|c|c|c|c|}
\hline Physical Fitness & BMI Groups & MEAN & SEM & P-Value \\
\hline \multirow{2}{*}{$\begin{array}{c}\text { Curl-up } \\
\text { (per minute) }\end{array}$} & Low & 29.68 & 0.67 & \multirow{2}{*}{$<0.0001$} \\
\hline & High & 15.76 & 0.99 & \\
\hline \multirow{2}{*}{$\begin{array}{l}\text { Sit \& Reach } \\
(\mathrm{cm})\end{array}$} & Low & 3.62 & 0.93 & \multirow{2}{*}{0.002} \\
\hline & High & -0.7 & 1.05 & \\
\hline \multirow{2}{*}{$\begin{array}{c}\text { Beep } \\
(\mathrm{m})\end{array}$} & Low & 3.68 & 0.22 & \multirow{2}{*}{$<0.001$} \\
\hline & High & 1.97 & 0.11 & \\
\hline
\end{tabular}

Table 3: Intergroup comparison of Low BMI and High BMI of school going children on various physical fitness components.

Significant $=p \leq 0.05$, Non-significant $=p>0.005$

\begin{tabular}{|c|c|c|c|c|}
\hline Physical Fitness & BMI Groups & MEAN & SEM & P-Value \\
\hline \multirow{2}{*}{$\begin{array}{c}\text { Curl-up } \\
\text { (per minute) }\end{array}$} & Low & 29.68 & 0.67 & \multirow{2}{*}{$<0.0001$} \\
\hline & Middle & 23.51 & 1.15 & \\
\hline \multirow{2}{*}{$\begin{array}{l}\text { Sit \& Reach } \\
\text { (cm) }\end{array}$} & Low & 3.62 & 0.93 & \multirow{2}{*}{$0.51^{*}$} \\
\hline & Middle & 4.42 & 1.35 & \\
\hline \multirow{2}{*}{$\begin{array}{c}\text { Beep } \\
(\mathrm{m})\end{array}$} & Low & 3.68 & 0.22 & \multirow{2}{*}{0.0003} \\
\hline & Middle & 2.78 & 0.14 & \\
\hline
\end{tabular}

Table 4: Intergroup comparison of Low BMI and Middle BMI of school going children on various physical fitness components.

Significant $=p \leq 0.05$, Non-significant $=p>0.005$ 


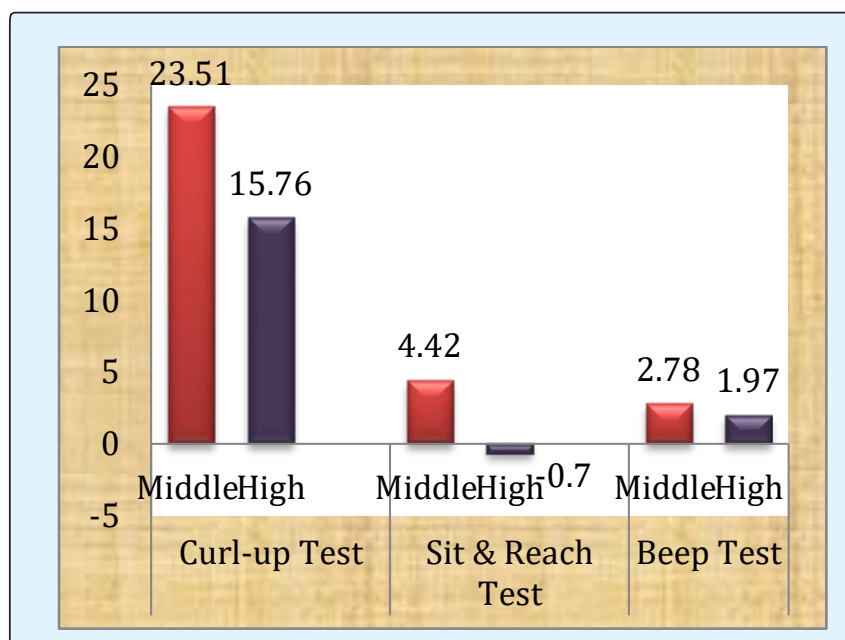

Figure 1: Comparison between middle \& high BMI groups.

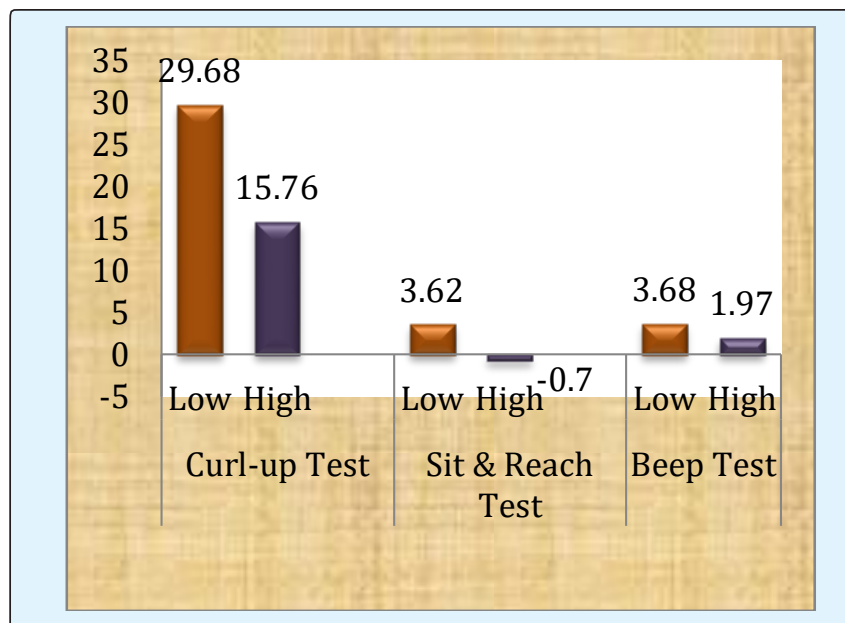

Figure 2: Comparison between low \& high BMI groups.

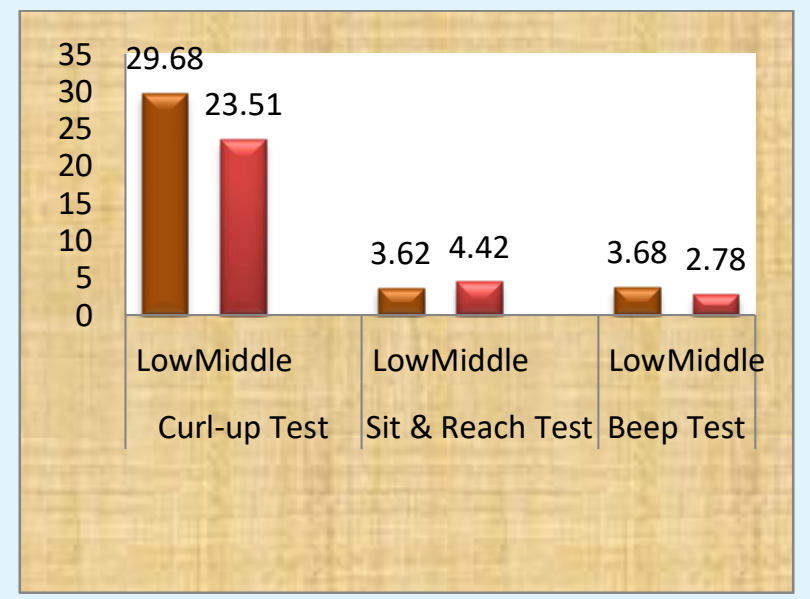

Figure 3: Comparison between low \& middle BMI groups.

\section{Discussion}

The aim of this study was to determine the relationship between physical fitness and BMI of school going children.

Findings of descriptive data of school going children belonging to $8^{\text {th }}$ and $9^{\text {th }}$ grade (Boys and Girls) of low BMI, middle BMI and high BMI on various physical test components indicated that difference exists among 3 different BMI groups. Children with low and middle BMI group performed better on physical test components compared to high BMI group. However significant higher activity levels were observed in low BMI group and middle BMI group than high BMI group.

The result of this study showed that low BMI group children having more muscular strength \& endurance level compared to middle BMI group children whereas in flexibility, both groups are somewhat equal. Middle BMI students showed more muscular strength, flexibility and endurance in comparison with high BMI students whereas students with low BMI showed high level of muscular strength \& endurance in comparison with middle BMI. Low BMI students showed high level of muscular strength, flexibility and endurance in comparison with high BMI students. The students with less BMI would show high physical fitness. High BMI students showed low level of muscular strength, flexibility and endurance in comparison with low BMI \& middle BMI group students.

Nowadays, children spend lot of time in using computers and playing during free period of time or even in their weekends instead of being active or performing outdoor activities or sports. Inactive lifestyle is responsible to cause many health related complications in later stage of life.

Staying active means keeping your body functioning at a high level. Regular exercise will maintain the performance of your lungs and heart to most efficiently burn off excess calories and keep your weight under control. Exercise will also improve muscle strength, increase joint flexibility and improve endurance. In general meaning, physical fitness is a general state of a good physical health. Obtaining a physical fitness is a result of certain great physical activity, proper nutrition and diet and most important is the proper course of physical recovery which is rest. In a nutshell, physical fitness is the fine- tuning of the human body to perform and work well. Another main benefit of physical activity is 


\section{Annals of Physiotherapy \& Occupational Therapy}

that it decreases the risk of heart disease. Additionally, it can decrease your risk of stroke, colon cancer, diabetes and high blood pressure.

Additionally there is a subsidiary recommendation for activities which help children to enhance muscular strength and flexibility.

\section{Limitations}

- Participants in this study attended the same school; thus, the population may be more homogenous.

- Participants represented a narrow age further limiting the generalizability of the findings.

- In sit \& reach test, score may be differ due to variations in arm, leg and trunk length, it can make comparisons between individuals misleading. This test is specific to the ROM, muscles \& joints of lower back and hamstring. It may not be relevant to other parts of the body.

- In beep test, practice \& motivation levels can influence the score attained. As the test is often conducted outside, the environmental conditions can affect the results.

\section{Conclusion}

This study concluded that low BMI group children \& middle BMI group children were found to be better in muscular strength, flexibility and endurance level compared to the high BMI group children.

\section{References}

1. Kraus H, Rabb W (1961) Hypokinetic Disease. Illinosis: Thomas Springfield.

2. Ng M, Fleming T, Robinson $M$, Thomson B, Hearts N, et al. (2014) Global, regional and National prevalence of overweight and obesity in children and adults during 1980-2013: a systematic analysis for the global burden of disease study 2013. Lancet 384(9945): 766-781.

3. Haslam DW, James WP (2005) Obesity. Lancet (Review). 366(9492): 1197-1209.

4. Bray GA (1985) Complications of obesity. J Med 103(6): 1052-1062

5. Yazdi FT, Clee SM, meyre D (2015) "Obesity genetics in mouse \& human: back \& forth, \& back again." Peer J 3: e856.

6. Nassis GP, Psarra G, Sidossis LS (2005) Central and total adiposity are lower in overweight and obese children with high cardiorespiratory fitness. Eur J Clin Nutr 59(1): 137-141.

7. Leger LA, Mercier D, Gadoury (1988) Validity of multistage 20-m shuttle run test 6(2): 93-101. 